\title{
Embolização da artéria gástrica esquerda como tratamento para a obesidade: revisão
} sistemática e metanálise

\author{
Left gastric artery embolization as a treatment for obesity: systematic review and meta-analysis \\ La embolización de la arteria gástrica izquierda como tratamiento para la obesidad: revisión
}

sistemática y meta-análisis

Recebido: 21/12/2020 | Revisado: 23/12/2020 | Aceito: 27/12/2020 | Publicado: 01/01/2021

\author{
Rafaela Aparecida Dias de Oliveira \\ ORCID: https://orcid.org/0000-0003-3399-311X \\ Universidade de Rio Verde, Brasil \\ E-mail: medicinarafaoliveira@gmail.com \\ Eduardo Teodoro Lima \\ ORCID: https://orcid.org/0000-0002-9796-2147 \\ Universidade Católica de Brasília, Brasil \\ E-mail: eteodoro155@gmail.com \\ Lívia Caetano da Silva Leão \\ ORCID: https://orcid.org/0000-0003-4462-327X \\ Faculdade UniBRAS Rio Verde, Brasil \\ E-mail: liviacsl@gmail.com \\ Marcelo Macedo Martins Neto \\ ORCID: https://orcid.org/0000-0002-8451-840X \\ Universidade de Rio Verde, Brasil \\ E-mail: martinsneto@unirv.edu.br
}

\begin{abstract}
Resumo
A embolização arterial gástrica (EAG) é uma das terapias minimamente invasivas que vêm sendo estudada como alternativa à cirurgia bariátrica, a fim de se mitigar possíveis complicações cirúrgicas. A EAG é realizada há mais de 40 anos para tratamento de hemorragias gastrointestinais, porém mais recentemente pesquisadores estudam a hipótese de que o procedimento possa ser realizado com finalidade anorexígena, associando a redução na produção do hormônio grelina à perda ponderal alcançada. Essa revisão sistemática objetiva sumarizar os resultados obtidos nos últimos 5 anos, avaliando a eficácia e segurança da EAG com finalidade anorexígena e sua relação com o hormônio grelina. Metodologia: Foi feita busca cruzada com os descritores "gastric artery embolization" e "obesity", retirados do DeCS/MeSH, nas bases de dados Pubmed, MEDLINE e Cochrane Library. Resultados: Essa revisão revelou 87 pacientes obesos submetidos à embolização da artéria gástrica esquerda, com perda ponderal média de 9,32\% $(\mathrm{T}=6,9$ meses) e redução média dos níveis séricos de grelina de 15,05\% ( $\mathrm{T}=6$ meses). Discussão: Até o momento, os resultados sugerem que a EAG pode ser uma técnica segura, porém não eficaz para o tratamento da obesidade. Considerações finais: Considerando-se que a cirurgia bariátrica proporciona perda de peso sustentada entre $45-80 \%$ em até 2 anos, são necessários ensaios com $\mathrm{N}$ maior para avaliação de perda ponderal sustentada proporcionalmente à queda dos níveis de grelina, pois estatisticamente a relação entre as variáveis não se comprova. Além disso, são necessárias pesquisas com maior duração para análise de potenciais complicações a longo prazo.
\end{abstract}

Palavras-chave: Artéria gástrica; Obesidade; Perda de peso; Embolização.

\begin{abstract}
Gastric artery embolization (GAE) is one of the minimally invasive therapies that have been studied as an alternative to bariatric surgery, in order to mitigate possible surgical complications. GAE has been performed for more than 40 years for the treatment of gastrointestinal hemorrhages, but more recently researchers have been studying the hypothesis that the procedure can be performed for anorexigenic purposes, associating the reduction in the production of the ghrelin hormone with the weight loss achieved. This systematic review aims to summarize the results obtained in the last 5 years, evaluating the efficacy and safety of GAE for anorexigenic purposes and its relation to the hormone ghrelin. Methodology: A cross-search was performed with the headings "gastric artery embolization" and "obesity", retrieved from DeCS / MeSH, in the Pubmed, MEDLINE and Cochrane Library databases. Results: This study revealed 87 obese patients undergoing embolization of the left gastric artery, with an average weight loss of $9.32 \%$ ( $T$ $=6.9$ months) and an average reduction in serum ghrelin levels of $15.05 \%$ ( $\mathrm{T}=6$ months). Discussion: So far, the results suggest that GAE may be a safe, but not effective, technique for the treatment of obesity. Final remarks: Considering that bariatric surgery provides sustained weight loss between $45-80 \%$ in up to 2 years, tests with higher $\mathrm{N}$ are needed to assess sustained weight loss proportionally to the drop in ghrelin levels, because the association between
\end{abstract}


these variables is not proven statistically. In addition, longer-term research is needed to analyze potential long-term complications.

Keywords: Gastric artery; Obesity; Weight loss; Embolization.

\section{Resumen}

La embolización de la arteria gástrica izquierda (EAGI) es una de las terapias mínimamente invasivas que se han estudiado como alternativa a la cirugía bariátrica. Se ha realizado durante más de 40 años para el tratamiento de las hemorragias gastrointestinales, pero más recentemente se ha estudiado la hipótesis de que el procedimiento puede utilizarse con fines anorexígenos, asociando la reducción de la grelina con la pérdida de peso lograda. Esta revisión sistemática apunta resumir los resultados obtenidos en los últimos 5 años, evaluando la eficacia y la seguridad del EAGI con fines anorexigénicos y su relación con la hormona grelina. Métodos: Se realizo búsqueda cruzada con "embolización de la arteria gástrica" y "obesidad", recuperados de DeCS / MeSH, en las bases de datos Pubmed, MEDLINE y Cochrane Library. Resultados: Este estudio reveló 87 pacientes obesos sometidos a EAGI, con una pérdida de peso promedio de 9,32\% ( $\mathrm{T}=6,9$ meses) y una reducción promedio de los niveles de grelina sérica de 15,05\% ( $\mathrm{T}=6$ meses). Discusión: Hasta ahora, los resultados sugieren que la GAE puede ser una técnica segura, pero no efectiva, para el tratamiento de la obesidad. Comentarios finales: Considerando que la cirugía proporciona una pérdida de peso sostenida de entre $45-80 \%$ en hasta 2 años, se necesitan pruebas con mayor N para evaluar la pérdida de peso sostenida proporcionalmente a la caída de los niveles de grelina, ya que la asociación entre estas variables no está probada estadísticamente. Además, se necesita investigar las complicaciones a largo plazo.

Palabras clave: Arteria gástrica; Obesidad; Reducción de peso; Embolización.

\section{Introdução}

Segundo dados do Observatório de Saúde Global da Organização Mundial da Saúde (OMS), a prevalência da obesidade praticamente triplicou nos últimos 40 anos. A OMS aponta que em 2016 mais de 650 milhões de adultos eram considerados obesos e quase 380 milhões de crianças e adolescentes estavam diagnosticados com sobrepeso ou obesidade. No Brasil, 22.1\% da população adulta têm obesidade (18.7-25.7), estando outros 56.5\% (52.3-60.7) com sobrepeso (World Health Organization, 2018).

Dado que mudança dos hábitos de vida e terapia farmacológica são muitas vezes ineficazes, cada dia mais pacientes são submetidos à cirurgia bariátrica. Porém, apesar das técnicas cirúrgicas menos invasivas que têm sido desenvolvidas, esse procedimento ainda pode incorrer em complicações como sangramento gastrointestinal, perfuração gástrica e obstrução intestinal (Abu Dayyeh et al., 2017; Neylan et al., 2016). A fim de mitigar tais efeitos, uma das abordagens que tem sido alvo de interesse como terapia minimamente invasiva é a embolização arterial gástrica (EAG), também chamada de embolização bariátrica.

A embolização é uma técnica guiada por imagem, que visa a oclusão endovascular intencional de artérias e veias. O método baseia-se no princípio de que havendo redução do suprimento sanguíneo no fundo gástrico, os níveis séricos de grelina serão reduzidos e, assim, o apetite será controlado (Weiss et al., 2015).

Desse modo, análoga à gastrectomia vertical, a embolização da artéria gástrica esquerda parece indiretamente afetar a produção de ácido gástrico e a motilidade e absorção gástricas, levando à perda de peso por mecanismos tanto endócrinos quanto fisiológicos (Bai et al., 2018; Anton et al., 2016).

De fato, estudos já demonstram que, dentre as mudanças metabólicas, a maior alteração após a cirurgia bariátrica se dá nas concentrações dos hormônios grelina, leptina, GLP-1 e PYY, sendo a grelina o hormônio comprovadamente orexígeno atuante no controle do apetite e da ingestão alimentar a curto prazo, bem como na regulação da glicose e no metabolismo energético (Camilleri, 2015).

A embolização da artéria gástrica esquerda é realizada há mais de 40 anos para tratamento de hemorragias gastrointestinais. Contudo, estudos com o intuito de avaliar a perda ponderal são mais incipientes e têm dividido opiniões em relação à validade do procedimento para esse fim. Essa revisão sistemática objetiva sumarizar os resultados obtidos nos artigos publicados nos últimos 5 anos, avaliando a eficácia e segurança da embolização da artéria gástrica esquerda com finalidade 
anorexígena e sua relação com o hormônio grelina.

\section{Metodologia}

Trata-se de uma revisão sistemática realizada segundo as recomendações do Preferred Reporting Items for Systematic Reviews and Meta-Analyses (PRISMA) (Moher, 2019) e do livro Metodologia da pesquisa científica (Pereira, A.S., 2018). A pesquisa é de natureza quali-quanti e foi realizada nas bases de dados Pubmed, MEDLINE, Cochrane Library e Scielo. Foi feita busca cruzada com os descritores "gastric artery embolization" e "obesity", retirados do Descritores em Ciências da Saúde/Medical Subject Headings (DeCS/MeSH). Por se tratar de um procedimento recente, para proporcionar revisão atual do tema e análise de resultados de pesquisas envolvendo seres humanos, incluíram-se artigos publicados entre 2015 e 2019, nos idiomas inglês e/ou português. A primeira triagem foi feita a partir da leitura dos títulos. Em seguida, foram lidos os resumos dos artigos. Como fatores de inclusão estão: trabalhos com o objetivo primário de avaliar a perda ponderal pós EAG, incluindo opinião de especialistas, estudos retrospectivos e prospectivos. Os trabalhos utilizados na metanálise foram aqueles que apresentaram coleta de dados em follow-up. Foram aplicados como critérios de exclusão: trabalhos anteriores a 2015 (2), artigos que tratassem da EAG para outros fins que não anorexígeno (5), estudos realizados em animais (3), artigos que não estivessem em inglês ou português (1). Um estudo anterior a 2015 foi incluído por ser considerado relevante, por ser o primeiro realizado em seres humanos a propor a relação aqui discutida e devido também ao número reduzido de trabalhos conduzidos em seres humanos até o momento. 25 dos artigos encontrados estavam em duplicidade e foram excluídos utilizando-se a ferramenta EndNote X9 ${ }^{\circledR}$. Por fim, restaram 26 artigos a serem lidos na íntegra. Desses, 10 foram incluídos na metanálise porque apresentavam dados originais. Os 16 trabalhos restantes eram revisões ou opiniões de especialistas e também foram considerados na discussão. O fluxograma metodológico está resumido na Figura 1. A avaliação crítica da qualidade dos trabalhos originais foi feita pelos autores seguindo as diretrizes do instrumento "APPRAISAL TOOL TO ASSESS THE QUALITY OF CROSS-SECTIONAL STUDIES (AXIS)". A análise dos dados encontrados nos artigos selecionados foi feita com o auxílio dos programas Excel @ e QtiPlot ® e suas ferramentas de análise estatística. As técnicas estatísticas utilizadas foram cálculos de média simples, ponderada e desvio padrão. No Excel @ foi feita uma tabulação cruzada dos dados que constam nas tabelas 1 e 2, e em seguida procedeu-se à análise estatística, fazendo-se os cálculos com o uso das funções MÉDIA, SOMA e SOMARPRODUTO, do Excel ${ }^{\circledR}$, comparando-se os dados obtidos de acordo com o número de pacientes de cada estudo (N) e com o tempo de follow-up. Para a construção dos gráficos foi utilizado o QtiPlot ® (Figuras 2, $3,4,5$ ). 
Figura 1: Fluxograma metodológico.
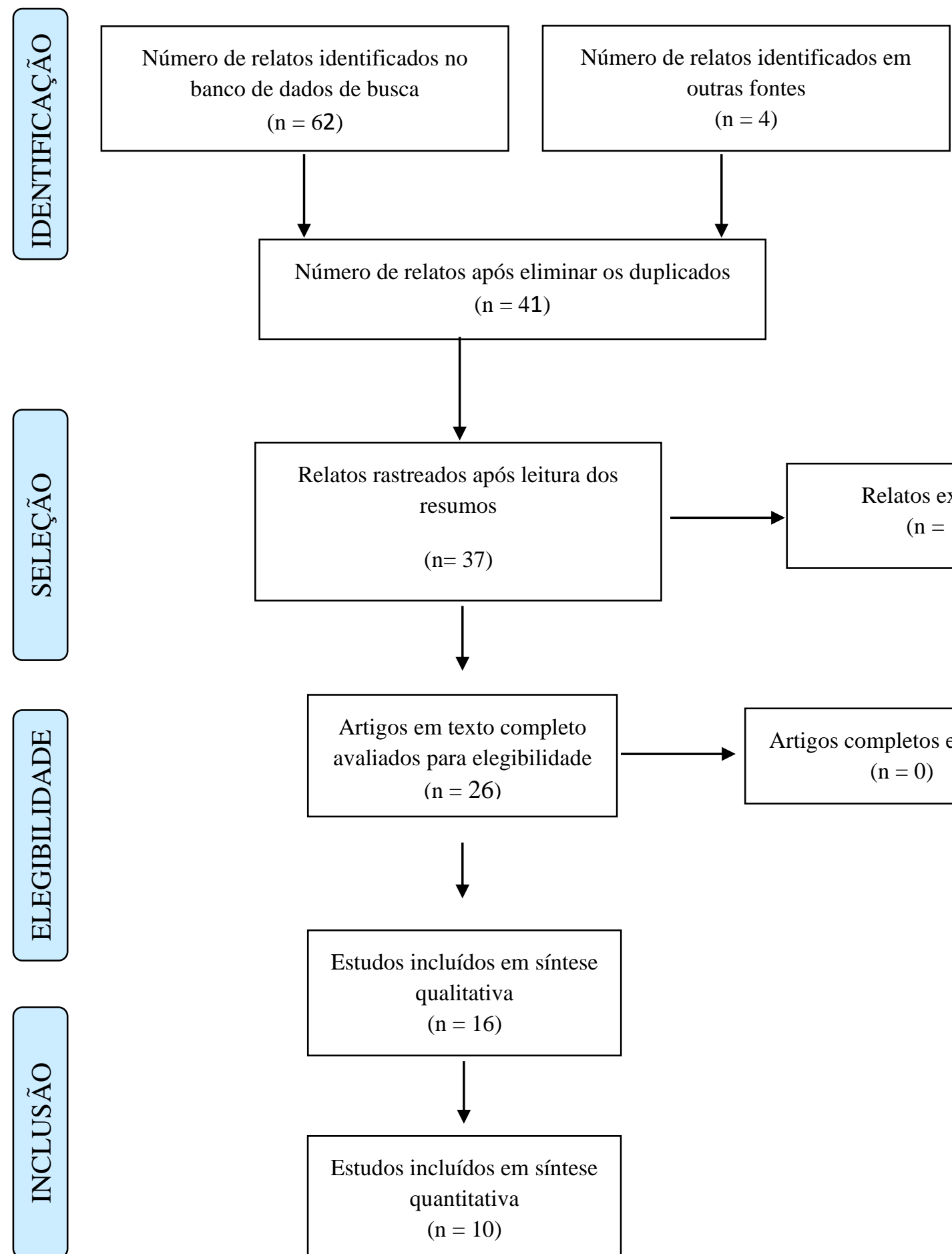

Fonte: Adaptado de PRISMA.

\section{Resultados e Discussão}

O número de artigos encontrados, por base de dados, foi: Pubmed (36 resultados), MEDLINE (22 resultados), Cochrane Library (4 resultados) e Scielo (0 resultado). Para análise estatística dos dados foram analisados todos os resultados publicados, ao nosso conhecimento, de ensaios clínicos e estudos retrospectivos que investigaram perda de peso após a embolização da artéria gástrica esquerda, totalizando 10 artigos, sendo 7 deles estudos prospectivos e 3 retrospectivos, como consta na tabela 1 . 
O tempo médio de follow-up foi 6,9 meses. Os materiais utilizados para embolização foram: Coils (platina), Gelfoam (gelatina absorvível), PVA - Polivinil-álcool, Bead Block (microesfera com base em PVA recoberta com hidrogel compressível), Embosphere (microesferas embolizantes não absorvíveis) e quimio-embolização. Dentre estes, os principais agentes embólicos utilizados foram agentes particulados, principalmente PVA, os quais mostraram maior porcentagem de redução dos níveis de grelina e perda ponderal (Gunn \& Oklu, 2014; Takahashi et al., 2019). 
Tabela 1: Estudos publicados avaliando embolização da artéria gástrica esquerda na perda de peso.

\begin{tabular}{|c|c|c|c|c|c|c|c|c|}
\hline Autores & Ano & Tipo do estudo & Material de embolização & $\mathrm{N}$ & $\begin{array}{c}\text { Redução dos Níveis de } \\
\text { Grelina }\end{array}$ & Perda ponderal média & $\begin{array}{c}\text { Em } T=x \\
\text { meses } \\
\text { (tempo de } \\
\text { follow up) }\end{array}$ & $\begin{array}{l}\text { Tempo total de } \\
\text { follow up do } \\
\text { estudo }\end{array}$ \\
\hline Gunn et al. & 2014 & Retrospectivo & $\begin{array}{c}\text { Coils (9) } \\
\text { Gelfoam (5) } \\
\text { PVA (5) }\end{array}$ & 19 & $\mathrm{NM}$ & $7,3 \%$ & $3 m$ & NSA \\
\hline $\begin{array}{l}\text { Kipshidze et } \\
\text { al. }\end{array}$ & 2015 & Prospectivo & $\begin{array}{l}300-500 \mu \mathrm{m} \\
\text { Bead Block }\end{array}$ & 5 & $19 \%$ & $16 \%$ & $6 \mathrm{~m}$ & $20-24 m$ \\
\hline $\begin{array}{l}\text { Salsamendi et } \\
\text { al. }\end{array}$ & 2015 & Retrospectivo & Quimio embolização & 1 & $\mathrm{NM}$ & $12 \%$ & $6 \mathrm{~m}$ & NSA \\
\hline Syed et al. & 2016 & Prospectivo & $\begin{array}{l}300-500 \mu \mathrm{m} \\
\text { Bead Block }\end{array}$ & 4 & Houve aumento de 5,3\% & $8,5 \%$ & $6 \mathrm{~m}$ & $6 \mathrm{~m}$ \\
\hline Weiss et al. & 2017 & Prospectivo & $\begin{array}{l}300-500 \mu \mathrm{m} \\
\text { Embospheres }\end{array}$ & 5 & $17,5 \%$ & $9 \%$ & $3 \mathrm{~m}$ & $6 \mathrm{~m}$ \\
\hline Bai et al. & 2018 & Prospectivo & $\begin{array}{c}\text { PVA } \\
500-710 \mu \mathrm{m}\end{array}$ & 5 & $24,82 \%$ & $12,64 \%$ & $9 \mathrm{~m}$ & $9 \mathrm{~m}$ \\
\hline Pirlet et al. & 2018 & Prospectivo & $\begin{array}{c}\text { PVA } \\
300-500 \mu \mathrm{m}\end{array}$ & 7 & $\mathrm{NM}$ & $4,77 \%$ & $2 \mathrm{~m}$ & $12 \mathrm{~m}$ \\
\hline Weiss et al. & 2019 & Prospectivo & $\begin{array}{l}300-500 \mu \mathrm{m} \\
\text { Embospheres }\end{array}$ & 15 & $\mathrm{NM}$ & $11,5 \%$ & $12 \mathrm{~m}$ & $12 \mathrm{~m}$ \\
\hline Zaitoun et al. & 2019 & Prospectivo & NM & 10 & NM & $8,9 \%$ & $6 \mathrm{~m}$ & $6 \mathrm{~m}$ \\
\hline $\begin{array}{l}\text { Takahashi et } \\
\text { al. }\end{array}$ & 2019 & Retrospectivo & $\mathrm{NM}$ & 16 & $\mathrm{NM}$ & $6,4 \%$ & $0,7-2,3 \mathrm{~m}$ & NSA \\
\hline
\end{tabular}

Fonte: Autores. 


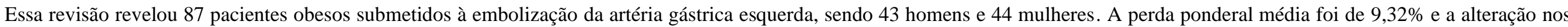

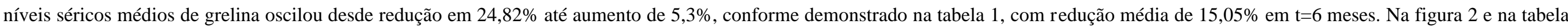
2 é possível ver a perda ponderal relatada em $1 ; 1,5 ; 2 ; 3 ; 6 ; 9 ;$ e 12 meses após a realização da embolização arterial gástrica.

Tabela 2: Estudos publicados avaliando a embolização arterial gástrica, mês a mês.

\begin{tabular}{|c|c|c|c|c|c|c|c|c|}
\hline Autores & $\mathrm{N}$ & 1 mês & 1,5 meses & 2 meses & 3 meses & 6 meses & 9 meses & 12 meses \\
\hline Gunn et al. 2014 & 19 & NM & NM & NM & $\mathrm{PP}=7,3 \%$ & NM & NM & NM \\
\hline $\begin{array}{l}\text { Kipshidze et al. } \\
2015\end{array}$ & 5 & $\begin{array}{c}\mathrm{PP}=10 \% \\
\mathrm{RG}=27,6 \%\end{array}$ & NM & $\mathrm{NM}$ & $\begin{array}{c}\mathrm{PP}=13 \% \\
\mathrm{RG}=33,33 \%\end{array}$ & $\begin{array}{c}\mathrm{PP}=16 \% \\
\mathrm{RG}=19,11 \%\end{array}$ & NM & $\mathrm{PP}=17 \%$ \\
\hline $\begin{array}{l}\text { Salsamendi et al. } \\
2015\end{array}$ & 1 & NM & NM & NM & NM & $\mathrm{PP}=12 \%$ & NM & NM \\
\hline Syed et al. 2016 & 4 & NM & NM & NM & NM & $\begin{array}{c}\mathrm{PP}=8,5 \% \\
\mathrm{RG}=\text { aumento de } 5,3 \%\end{array}$ & NM & NM \\
\hline Weiss et al. 2017 & 5 & NM & NM & NM & $\mathrm{RG}=17,5 \%$ & NM & NM & NM \\
\hline Bai et al. 2018 & 5 & NM & NM & NM & $\begin{array}{c}\mathrm{PP}=7,58 \% \\
\mathrm{RG}=40,83 \%\end{array}$ & $\begin{aligned} \mathrm{PP} & =9,79 \% \\
\mathrm{RG} & =31,94 \%\end{aligned}$ & $\begin{array}{l}\mathrm{PP}=12,64 \% \\
\mathrm{RG}=24,82 \%\end{array}$ & NM \\
\hline Pirlet et al. 2018 & 7 & NM & NM & $\mathrm{PP}=4,38 \%$ & NM & $\mathrm{PP}=3,75 \%$ & NM & $\mathrm{PP}=8,125 \%$ \\
\hline Weiss et al. 2019 & 15 & $\mathrm{PP}=8,2 \%$ & NM & NM & $\mathrm{PP}=11,2 \%$ & $\mathrm{PP}=12,8 \%$ & NM & $\mathrm{PP}=11,5 \%$ \\
\hline Zaitoun et al. 2019 & 10 & NM & NM & NM & NM & $\mathrm{PP}=8,9 \%$ & NM & NM \\
\hline $\begin{array}{c}\text { Takahashi et al. } \\
2019\end{array}$ & 16 & NM & $\mathrm{PP}=6,4 \%$ & NM & NM & NM & NM & NM \\
\hline
\end{tabular}


Dos 87 pacientes, 19 foram embolizados para controle de hemorragia gastrointestinal, sendo retrospectivamente avaliados quanto à perda ponderal no estudo de Gunn e Oklu (2014), o primeiro estudo a propor a relação entre a embolização da artéria gástrica esquerda e a perda de peso (Gunn \& Oklu, 2014).

De acordo com a Figura 2, percebe-se que ao longo do tempo há um padrão crescente de perda de peso após a embolização, que pode ser observado pela curva em vermelho, a qual corresponde à regressão polinomial da perda de peso observada nos ensaios clínicos.

Figura 2: Perda ponderal (\%) ao longo de 12 meses.

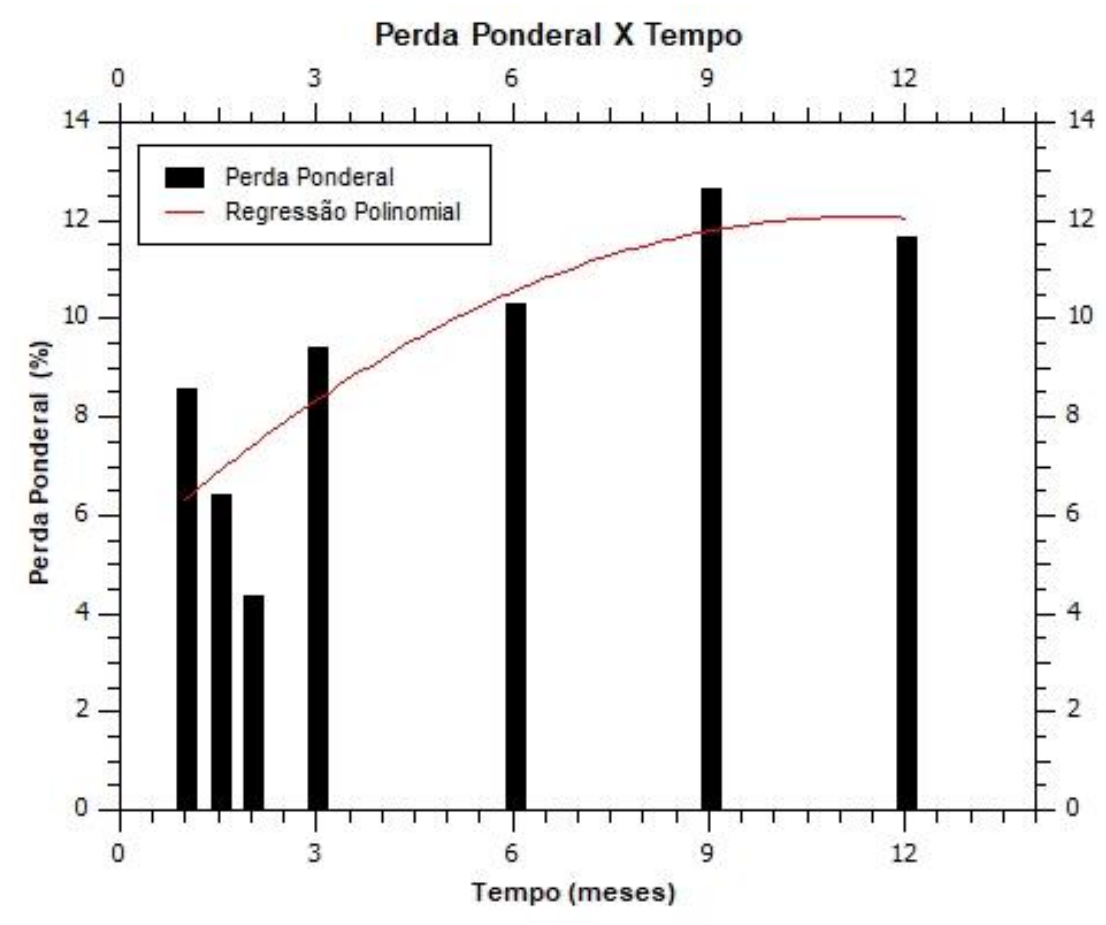

Fonte: Autores.

A redução observada é de aproximadamente $6 \%$ do peso inicial, a partir do $1^{\circ}$ mês, e há aumento progressivo dessa perda ponderal, até atingir o máximo de aproximadamente $12 \%$ no $12^{\circ}$ mês de acompanhamento.

Em revisão sistemática publicada em 2016, de um total de 67 pacientes, dos quais 56 eram obesos e 11 obesos mórbidos, houve redução no índice de massa corporal (IMC) de, respectivamente, 7\% e 11\%, durante os 3 primeiros meses de pós-operatório, acompanhada de redução da concentração de grelina de 17,5\% \pm 29 (Carvalho et al., 2018). Uma outra revisão sistemática, publicada em 2018, resultou em análise de 62 pacientes submetidos à embolização. No primeiro trimestre após o procedimento, houve redução ponderal entre 7-11\% e diminuição no nível de grelina em 36\% (Kordzadeh et al., 2018). Com resultados semelhantes, a metanálise mais recente publicada, com dados de 47 pacientes, revela perda ponderal de $8,85 \mathrm{~kg} \pm$ 1,24 ( $<$ 0,001) após 12 meses de seguimento pós-operatório (Hafezi-Nejad et al., 2019). Há ainda indicativos de que a EAG também possa ser um procedimento efetivo para pacientes pré-diabéticos [HbA1c variando de $6 \pm 0.2$ a $4.7 \pm 0.6$ em 6 meses ( $\mathrm{p}<0,0001)$ ], além de melhorar parâmetros de qualidade de vida, avaliados por meio do Short Form-36 Health Survey (SF-36) (Weiss et al., 2019; Zaitoun et al., 2019).

Dados preliminares de ensaios clínicos sugerem que a perda de peso alcançada pode estar relacionada com a diminuição da grelina. Contudo, os resultados ainda são controversos. Bai et al. (2018), em acompanhamento de 5 pacientes durante 9 meses, observou que os níveis de grelina aumentaram gradualmente nos 6 meses após a cirurgia e, mesmo com redução aos 9 meses, não houve diferença significativa entre os níveis basal, do $6^{\circ}$ mês e do $9^{\circ}$ mês. No ensaio GET LEAN, em 
$\mathrm{t}=6$ meses, em 2 pacientes os valores de grelina caíram e nos outros 2, sofreram aumento. Curiosamente, o paciente com maior redução de peso foi um dos que não apresentou redução de grelina (Syed et al., 2016). Como pode ser visto no gráfico da Figura 3, a análise feita dos dados levantados pelos estudos de Kipshidze et al. (2015), Syed et al. (2016) e Weiss et al. (2019) também confirmam esse aumento dos níveis de grelina a partir do $6^{\circ}$ mês.

Figura 3: Redução de grelina (\%) ao longo de 9 meses.

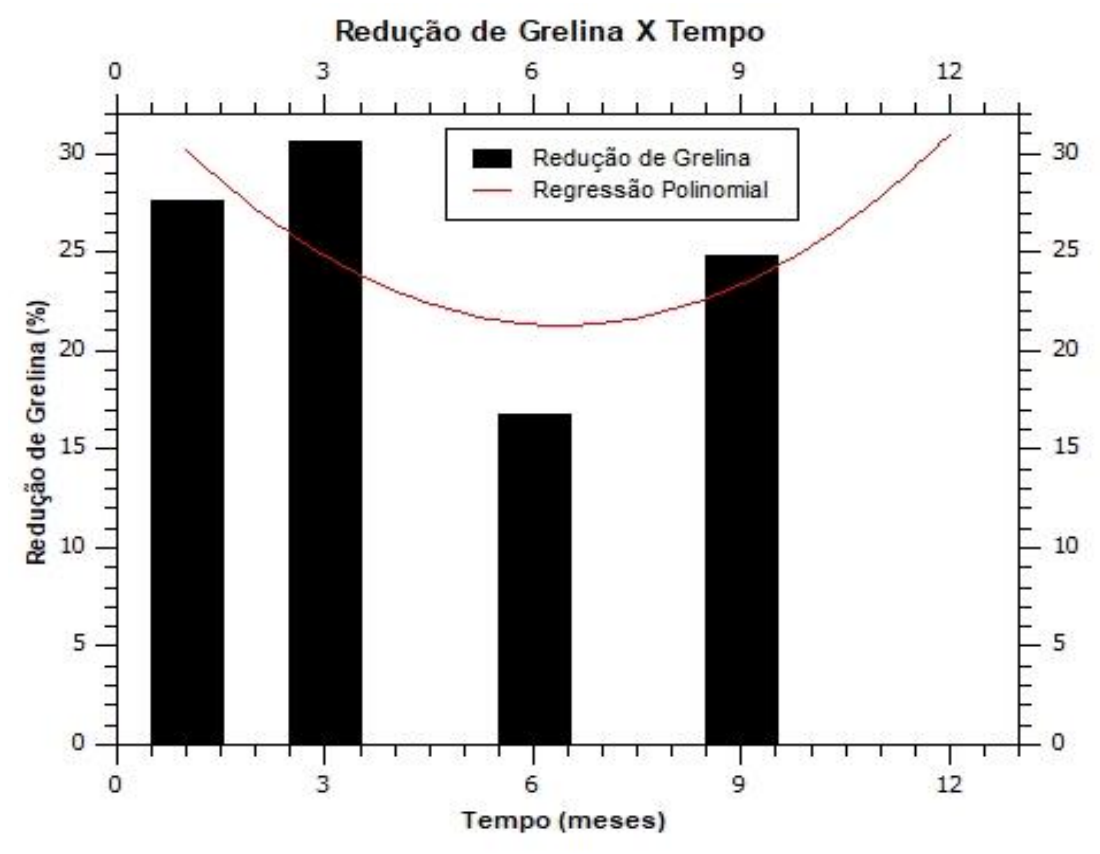

Fonte: Autores.

Algumas possíveis justificativas levantadas para os casos em que a grelina não reduz são: formação de circulações colaterais no fundo gástrico, maior produção de grelina pelos sítios "menores" como pâncreas e cólon e flutuação nas concentrações desse hormônio no sangue durante o dia, porquanto o nível poderia variar dependendo do horário em que o sangue foi coletado para análise (Syed et al., 2016; Bai et al., 2018).

É possível verificar ainda, no gráfico da Figura 4, que, com os dados até agora publicados, não é possível relacionar a redução dos níveis de grelina com a perda ponderal. 
Figura 4: Comparação entre perda ponderal e redução de grelina demonstrando que não parece haver relação direta entre as variáveis.

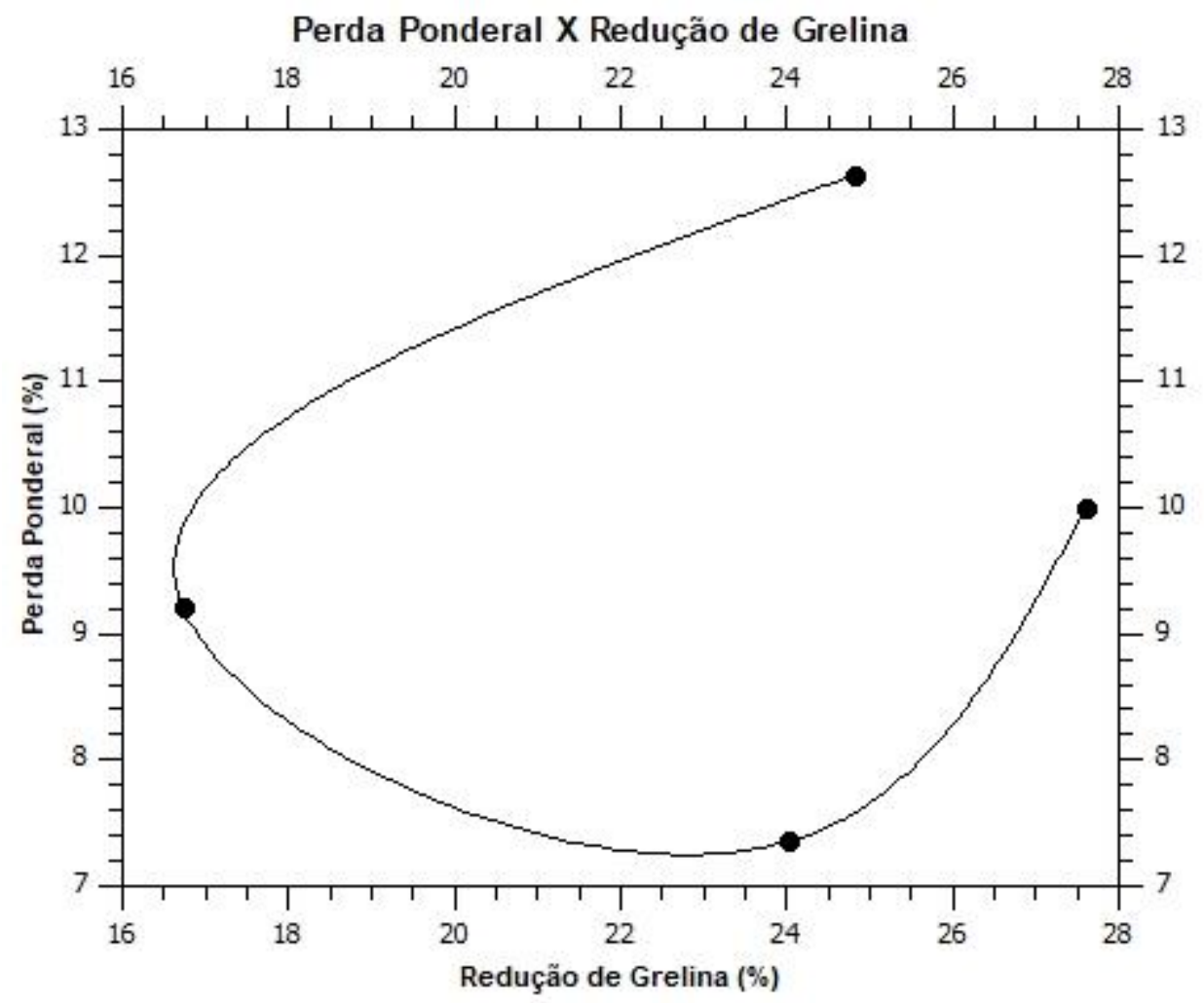

Fonte: Autores.

Observa-se que a perda ponderal não varia em função da redução de grelina. Caso a perda ponderal alcançada fosse consequência da redução dos níveis de grelina, a curva do gráfico se comportaria como uma função matemática conhecida. Assim, ainda são necessários mais estudos e análises com espaço amostral maior para investigar se existe realmente relação entre a redução de grelina e a perda ponderal atingida pelos pacientes. Possa ser que, mesmo sem a tendência de relação direta entre as variáveis perda ponderal e redução de grelina, a embolização seja eficaz para a perda de peso por outras razões.

Vale notar também a comparação entre a perda ponderal revelada nos estudos que registraram mudanças nos níveis de grelina com a perda ponderal dos estudos que não registraram os níveis do hormônio (Figura 5). 
Figura 5: Comparação de perda ponderal entre pacientes avaliados e não avaliados quanto à redução de grelina.

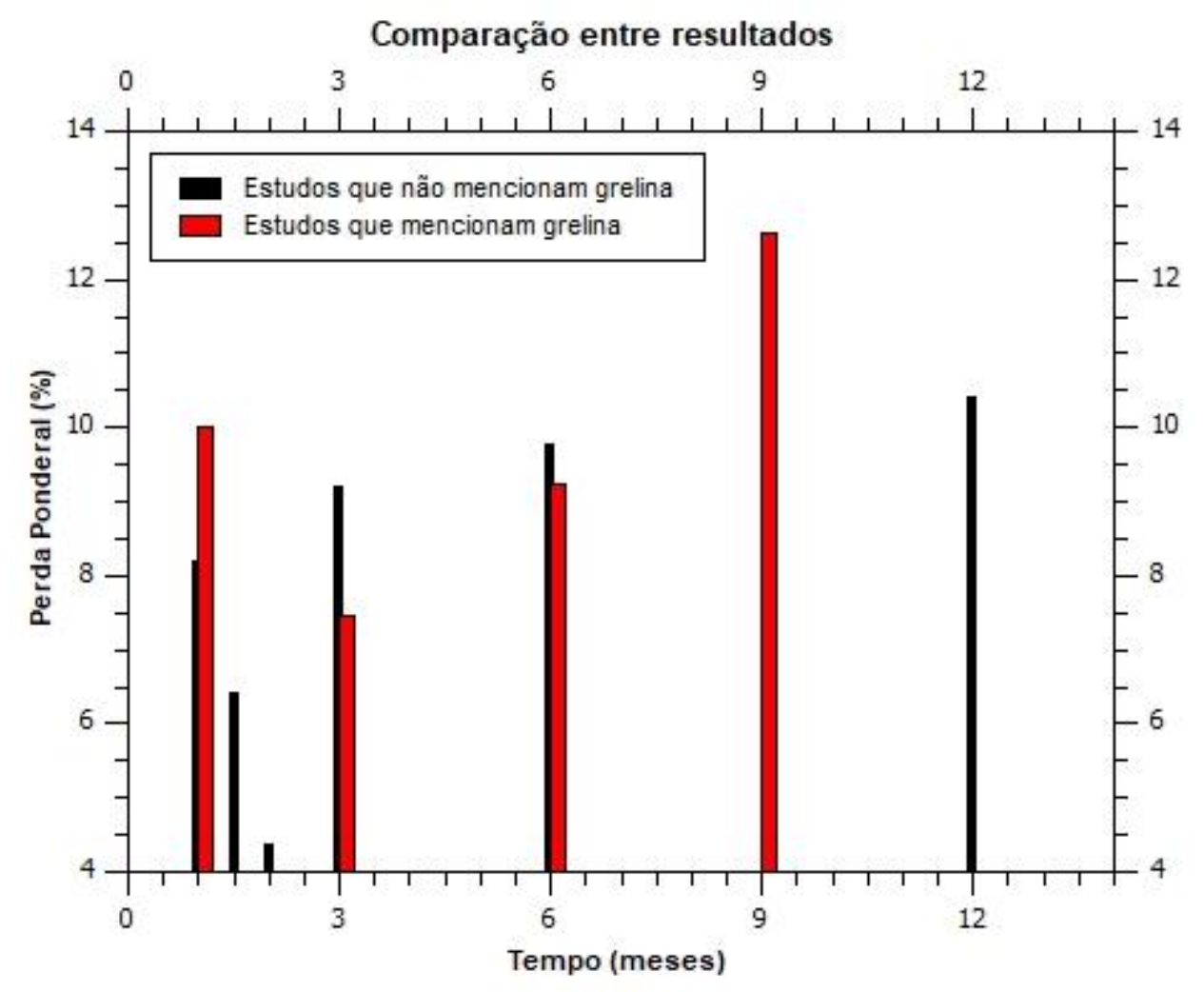

Fonte: Autores.

Nota-se que em ambos os grupos há perda ponderal semelhante. Mais uma vez os dados indicam que a perda ponderal realmente ocorre após a embolização da artéria gástrica esquerda. Entretanto, a hipótese levantada para explicar o efeito ainda não pode ser comprovada. Na faixa dos 6 meses, que é o último mês em que os estudos revelam tanto os resultados da perda de peso quanto os níveis de grelina, a perda ponderal está entre 9\% e 10\% (Kipshidze et al., 2015; Syed et al., 2016; Bai et al., 2018). É importante reiterar que esses resultados possuem espaço amostral muito pequeno, e que esse é o motivo da existência da grande discrepância de valores em alguns meses, além das diferenças entre os protocolos seguidos por cada pesquisador, como material utilizado e características dos pacientes incluídos no estudo.

Um dos argumentos levantados a favor da embolização da artéria gástrica esquerda em seres humanos é que a análise imunohistoquímica da mucosa gástrica e duodenal de modelos animais demonstra que o número de células produtoras de grelina diminui significativamente após a embolização da artéria gástrica esquerda (Weiss et al., 2015). Contudo, também não é possível generalizar tais resultados, pois esses estudos foram conduzidos em suínos e nesses animais a perfusão do fundo gástrico não provém como nos humanos da artéria gástrica esquerda, e sim principalmente das artérias gastroepiplóicas (Weiss \& Kathait, 2017).

Como os ensaios clínicos com resultados disponíveis estabeleceram diferentes critérios de inclusão aos participantes, o paciente ideal à EAG ainda não foi definido. Alguns especialistas sugerem que a EAG possa ser mais benéfica àqueles pacientes com sobrepeso moderado e níveis basais maiores de grelina, em comparação àqueles com obesidade mórbida que tenham níveis basais menores desse hormônio, ou em casos nos quais o paciente não seja candidato à cirurgia bariátrica no momento, mas tenha potencial de se beneficiar dela no futuro com a redução de peso alcançada pela embolização arterial gástrica (Angrisani et al., 2018; Gilchrist, 2019). 
Esse também é um aspecto controverso, pois alguns autores levantam a hipótese de que a EAG possa na verdade inutilizar a artéria gástrica esquerda (AGE), impossibilitando uma nova operação gástrica no futuro, como a bariátrica (Fink et al., 2018). Acreditamos que essa seja uma possibilidade real em pacientes que não alcancem perda ponderal desejada com esse procedimento e optem pela cirurgia. Dado que em um futuro bypass gástrico em "Y de Roux", por exemplo, a irrigação do pouch gástrico se tornaria dependente de vasos esofágicos distais e ramos da artéria gástrica esquerda, alterações isquêmicas poderiam ocorrer e evoluir com complicações locais (estenoses, fístulas, necrose).

Em relação à técnica mais adequada para a embolização, os ensaios clínicos também aplicaram abordagens distintas. No GET LEAN, por exemplo, todos os ramos distais da artéria gástrica esquerda foram embolizados, já no BEAT Obesity, todas as artérias que irrigam o fundo gástrico foram incluídas e no ensaio de Bai et al., apenas ramos selecionados da artéria gástrica esquerda foram utilizados para a embolização. O impacto dessas diferenças de abordagem ainda não foi investigado (Zhong et al., 2018).

No que diz respeito à via de acesso utilizada, a mais comum é a femoral, mas em estudo prospectivo, Pirlet et al. analisou 47 pacientes submetidos à embolização por acesso via radial e os resultados demonstram ser uma abordagem segura e viável, com a vantagem de o paciente poder deambular mais precocemente comparado ao procedimento realizado via femoral (Pirlet et al., 2018).

Até o momento nenhum estudo reportou efeitos adversos graves no pós operatório. O BEAT Obesity, um dos principais ensaios sendo desenvolvidos, observou apenas efeitos menores como náuseas, desconforto epigástrico, retardo do tempo de esvaziamento gástrico e ulcerações que desapareceram em 3 meses (Hafezi-Nejad et al., 2019; Shoar et al., 2016; Weiss et al., 2016).

Reconhecemos como fatores limitadores da nossa pesquisa: pequena amostra populacional, estudos retrospectivos, os quais foram inconclusivos quanto à redução dos níveis de grelina, estudos com outras variáveis que podem interferir na perda de peso, como a presença de câncer, além da quantidade limitada de estudos prospectivos, o que dificulta a generalização das estatísticas.

\section{Considerações Finais}

Os resultados sugerem que a EAG pode ser uma técnica segura, porém até o momento os dados não demonstram uma perda de peso significativa para ser considerada uma alternativa eficaz para o tratamento da obesidade. A hipótese até então advogada de que essa perda de peso é resultado da redução do hormônio grelina ainda não pode ser comprovada.

Considerando-se que a cirurgia bariátrica proporciona perda de peso sustentada entre 45-80\% em até 2 anos, sugerimos ensaios clínicos com $\mathrm{N}$ maior para avaliação de perda ponderal sustentada proporcionalmente à queda dos níveis de grelina para legitimar essa relação, além de pesquisas com maior duração para análise de potenciais complicações a longo prazo. Além disso, horários padronizados de coleta de sangue para análise dos níveis do hormônio grelina podem auxiliar na avaliação, ao evitar as variações do hormônio que ocorrem durante o dia.

Não obstante, deve-se reconhecer a redução de peso observada até o momento nos estudos, bem como a limitação dos efeitos adversos que a técnica propicia, o que na prática pode influenciar na decisão do paciente.

\section{Referências}

Abu Dayyeh, B. K., Edmundowicz, S., \& Thompson, C. C. (2017). Clinical practice update: Expert review on endoscopic bariatric therapies. Abu Dayyeh, B. K., Edmundowicz, S., \& Thompson, C. C. (2017). Clinical practice update: Expert review on endoscopic bariatric therapies. Gastroenterology, 152(4), 716729. https://doi.org/10.1053/j.gastro.2017.01.035

Angrisani, L., Santonicola, A., Vitiello, A., Ferraro, L., \& Iovino, P. (2018). Reply to letter to the editor "Left gastric artery Embolization for weight loss-a dead-end procedure". Obesity Surgery, 28(11), 3627-3628. https://doi.org/10.1007/s11695-018-3464-9. 
Anton, K., Rahman, T., Bhanushali, A., \& Patel, A. A. (2016). Bariatric left gastric artery Embolization for the treatment of obesity: A review of gut hormone involvement in energy homeostasis. American Journal of Roentgenology, 206(1), 202-210. https://doi.org/10.2214/ajr.15.14331.

Bai, Z., Qin, Y., Deng, G., Zhao, G., Zhong, B., \& Teng, G. (2018). Bariatric Embolization of the left gastric arteries for the treatment of obesity: 9-Month data in 5 patients. Obesity Surgery, 28(4), 907-915. https://doi.org/10.1007/s11695-017-2979-9.

Bai, Z., Zhong, B., \& Teng, G. (2018). Response to letter of "Left gastric artery Embolization for weight loss-a Deadend procedure". Obesity Surgery, 28(11), 3625-3626. https://doi.org/10.1007/s11695-018-3428-0.

Camilleri, M. (2015). Peripheral mechanisms in appetite regulation. Gastroenterology, 148(6), 1219-1233. https://doi.org/10.1053/j.gastro.2014.09.016.

Carvalho, M. N., Moura Filho, E. P., Silva, I. M. O., Souza, L. K. M. (2018). Embolização de ramos da artéria gástrica esquerda como tratamento neuroendócrino para obesidade: uma revisão sistemática [Poster session]. $1^{\mathrm{a}}$ Jornada Acadêmica de Medicina, Instituto de Educação Superior do Vale do Parnaíba - IESVAP

Clarivate Analytics. (2019). EndNote ®, Reference Management Software. https://www.endnote.com/?_ga=2.109951301.1174758763.160883120997773908.1607451281

Fink, J. M., Martini, V., Seifert, G., \& Marjanovic, G. (2018). Left gastric artery Embolization for weight loss-a dead-end procedure. Obesity Surgery, 28(11), 3623-3624. https://doi.org/10.1007/s11695-018-3427-1.

Gilchrist, I. C. (2019). A catheter-based bariatric procedure: Wishful thinking or an intriguing concept. Catheterization and Cardiovascular Interventions, 93(3), 371-372. https://doi.org/10.1002/ccd.28139.

Gunn, A. J., \& Oklu, R. (2014). A preliminary observation of weight loss following left gastric artery Embolization in humans. Journal of Obesity, 2014, 1-4. https://doi.org/10.1155/2014/185349.

Hafezi-Nejad, N., Bailey, C. R., Gunn, A. J., \& Weiss, C. R. (2019). Weight loss after left gastric artery Embolization: A systematic review and meta-analysis. Journal of Vascular and Interventional Radiology, 30(10), 1593-1603.e3. https://doi.org/10.1016/j.jvir.2019.06.020.

IONDEV SRL. (2013). QTIPLOT ® - Data Analysis and Scientific Visualization. https://www.qtiplot.com/index.html

Kipshidze, N., Archvadze, A., Bertog, S., Leon, M. B., \& Sievert, H. (2015). Endovascular bariatrics. JACC: Cardiovascular Interventions, 8(12), 1641-1644. https://doi.org/10.1016/j.jcin.2015.07.016.

Kordzadeh, A., Lorenzi, B., Hanif, M. A., \& Charalabopoulos, A. (2018). Left gastric artery Embolisation for the treatment of obesity: A systematic review. Obesity Surgery, 28(6), 1797-1802. https://doi.org/10.1007/s11695-018-3211-2.

Moher, D. (2009). Preferred reporting items for systematic reviews and meta-analyses: The PRISMA statement. Annals of Internal Medicine, 151(4), 264. https://doi.org/10.7326/0003-4819-151-4-200908180-00135

Neylan, C. J., Dempsey, D. T., Tewksbury, C. M., Williams, N. N., \& Dumon, K. R. (2016). Endoscopic treatments of obesity: A comprehensive review. Surgery for Obesity and Related Diseases, 12(5), 1108-1115. https://doi.org/10.1016/j.soard.2016.02.006.

Noncommunicable diseases country profiles 2018 (2018). Geneva: World Health Organization. License: CC BY-NC-SA 3.0 IGO.

Pereira, A. S., Shitsuka, D. M., Parreira, F. J., \& Shitsuka, R. (2018). Metodologia da pesquisa científica. NTE. https://repositorio.ufsm.br/bitstrea m/handle/1 /15824/Lic_Computacao_Metodologia-Pesquisa-Cientifica.pdf?sequence=1

Pereira, K., Salsamendi, J., Kang, K., \& Fan, J. (2015). Minimally invasive percutaneous endovascular therapies in the management of complications of nonalcoholic fatty liver disease (NAFLD): A case report. Journal of Radiology Case Reports, 9(9), 36-43. https://doi.org/10.3941/jrcr.v9i9.2557.

Pirlet, C., Ruzsa, Z., Costerousse, O., Nemes, B., Merkely, B., Poirier, P., \& Bertrand, O. F. (2018). Transradial left gastric artery embolization to treat severe obesity: A pilot study. Catheterization and Cardiovascular Interventions, 93(3), 365-370. https://doi.org/10.1002/ccd.27846.

Shoar, S., Saber, A. A., Aladdin, M., Bashah, M. M., AlKuwari, M. J., Rizwan, M., \& Rosenthal, R. J. (2016). Bariatric manipulation of gastric arteries: A systematic review on the potential concept for treatment of obesity. International Journal of Surgery, 36, 177-182. https://doi.org/10.1016/j.ijsu.2016.10.014.

Syed, M. I., Morar, K., Shaikh, A., Craig, P., Khan, O., Patel, S., \& Khabiri, H. (2016). Gastric artery Embolization trial for the lessening of appetite Nonsurgically (GET LEAN): Six-month preliminary data. Journal of Vascular and Interventional Radiology, 27(10), 1502-1508. https://doi.org/10.1016/j.jvir.2016.07.010.

Takahashi, E. A., Takahashi, N., Reisenauer, C. J., Moynagh, M. R., \& Misra, S. (2019). Body composition changes after left gastric artery embolization in overweight and obese individuals. Abdominal Radiology, 44(7), 2627-2631. https://doi.org/10.1007/s00261-019-02002-6.

Weiss, C. R., Abiola, G. O., Fischman, A. M., Cheskin, L. J., Vairavamurthy, J., Holly, B. P., Akinwande, O., Nwoke, F., Paudel, K., Belmustakov, S., Hong, K., Patel, R. S., Shin, E. J., Steele, K. E., Moran, T. H., Thompson, R. E., Dunklin, T., Ziessman, H., Kraitchman, D. L., \& Arepally, A. (2019). Bariatric Embolization of arteries for the treatment of obesity (BEAT obesity) trial: Results at 1 year. Radiology, 291(3), 792-800. https://doi.org/10.1148/radiol.2019182354.

Weiss, C. R., Akinwande, O., Paudel, K., Cheskin, L., Holly, B., Hong, K., Fischman, A. M., Patel, R. S., Shin, E., Steele, K., Moran, T. H., Kaiser, K., Shade, D., Kraitchman, D. L., \& Arepally, A. (2016). Bariatric Embolization of Arteries for the Treatment of Obesity (BEAT Obesity) Clinical Trial: 6-month Safety, Feasibility and Early Efficacy. https://www.hopkinsmedicine.org/institute_basic_biomedical_sciences/news_events/boot_camp/2016_weighty_matters /cliff\%20weiss\%20presentation. 
Research, Society and Development, v. 10, n. 1, e1510111415, 2021

(CC BY 4.0) | ISSN 2525-3409 | DOI: http://dx.doi.org/10.33448/rsd-v9i12.11415

Weiss, C. R., Gunn, A. J., Kim, C. Y., Paxton, B. E., Kraitchman, D. L., \& Arepally, A. (2015). Bariatric Embolization of the gastric arteries for the treatment of obesity. Journal of Vascular and Interventional Radiology, 26(5), 613-624. https://doi.org/10.1016/j.jvir.2015.01.017.

Weiss, C. R., \& Kathait, A. S. (2017). Bariatric embolization: A new and effective option for the obese patient? Expert Review of Gastroenterology \& Hepatology, 11(4), 293-302. https://doi.org/10.1080/17474124.2017.1294060.

Zaitoun, M. M., Basha, M. A., Hassan, F., Elsayed, S. B., Farag, A. A., Amer, M., Aly, S. A., \& Zaitoun, N. (2019). Left gastric artery Embolization in obese, Prediabetic patients: A pilot study. Journal of Vascular and Interventional Radiology, 30(6), 790-796. https://doi.org/10.1016/j.jvir.2019.02.010.

Zhong, B., Abiola, G., \& Weiss, C. R. (2018). Bariatric arterial Embolization for obesity: A review of early clinical evidence. Cardiovascular and Interventional Radiology, 41(11), 1639-1647. https://doi.org/10.1007/s00270-018-1996-y. 